\title{
Requerimiento Hídrico de Gmelina arborea en Etapa de Vivero Bajo Condiciones Controladas
}

\author{
Amir Vergara ${ }^{1}$, Teobaldis Mercado $^{1}$, Alfredo Jarma ${ }^{1}$, Kellen Gatti ${ }^{1}$ \\ ${ }^{1}$ Facultad de Ciencias Agricolas, Universidad de Córdoba, Montería, Córdoba, Colombia
}

\section{RESUMEN}

Con el fin de conocer el requerimiento hídrico de Melina en la etapa de vivero, se realizó un ensayo con cuatro coeficientes de consumo (Kc: $0,6,0,8,1,2,1,4$. Estos tratamientos se transformaron en láminas de riego de 2,4 mm.día ${ }^{-1}$, 3,2 mm.día ${ }^{-1}$, 4,8 mm.día ${ }^{-1}$ y 5,6 mm.día ${ }^{-1}$ respectivamente, debido a que la evapotranspiración de referencia (ETO) de la zona fue de $4 \mathrm{~mm}$.día ${ }^{-1}$. Se observó que a medida que se aumentaba la lámina de riego, disminuía el crecimiento de las plantas. Los valores más altos de altura de planta, área foliar, masa seca de hoja, masa seca del tallo y masa seca de raíz se obtuvieron con la lámina de 2,4 mm.día ${ }^{-1}$. Por otro lado, los índices de crecimiento (TAC, TRC, AFE, RAF) muestran la misma tendencia. Con base en estos resultados se determinó que el Kc para esta especie en la etapa de vivero es de 0,6.

Palabras clave: láminas de riego, coeficiente de cultivo (Kc), crecimiento.

\section{Water Requirement of Gmelina arborea on Nursery Stage Under Controlled Conditions}

\begin{abstract}
In order to recognize the Melina water requirement in the nursery stage, we performed a test with four crop coefficient (Kc: $0,6,0,8,1,2,1,4)$. These treatments included irrigation blades of $2,4 \mathrm{~mm} \cdot$ day $^{-1}, 3,2 \mathrm{~mm} \cdot$ day $^{-1}, 4,8 \mathrm{~mm}$.day ${ }^{-1}$ and $5.6 \mathrm{~mm}$. day ${ }^{-1}$ respectively, due to the area reference evapotranspiration at $4 \mathrm{~mm} \cdot$ day $^{-1}$. It was observed a decrease of plant growth as the raising of the irrigation blade. The highest values of plant height, leaf area, leaf dry weight, dry weight of stem and root dry weight were obtained with the blade of $2,4 \mathrm{~mm}$.day ${ }^{-1}$. On the other hand, the growth rates studied have shown the same trend. Based on these results we determined that the $\mathrm{Kc}$ for this species in the pond stage is 0,6 .
\end{abstract}

Keywords: irrigation levels, coefficient of cultivation (KC), growth. 


\section{INTRODUCCION}

En Colombia existen 25 millones de hectáreas de alto potencial para ser aprovechadas en cultivos forestales (Gatti et al., 2012), siendo el departamento de Córdoba el que dispone de 897.086,72 ha (Murillo et al., 2012). Sin embargo, a pesar de todas las ventajas comparativas mencionadas, es un importador de madera y procesados madereros. Melina (Gmelina arborea Roxb) es una especie forestal, originaria del Sureste Asiático y en la actualidad es uno de los maderables más utilizado para reforestar en la región del Caribe colombiano (Mariño \& Rodríguez, 2010).

Prieto et al. (2007) afirma que, para establecer un sistema de producción forestal, es necesario conocer de forma precisa todos los aspectos referentes al desarrollo de la planta, aspectos que van desde las fases iniciales hasta la siembra en campo. La calidad de las plántulas en los viveros se ve afectada, principalmente, por los siguientes factores: los materiales genéticos, los manejos hídricos y nutricionales, y la composición de los sustratos (Gomes, 2013; Silva et al., 2012). Sin embargo, de entre los recursos que la planta necesita para su buen desarrollo, el agua es el recurso más abundante y, a su vez, el más limitante para la productividad (Gomes, 2013; Taiz \& Zeiger, 2004); esto se debe a que, en la mayoría de los viveros, el manejo hídrico solamente se determina a través de un examen observacional de las plántulas, por lo que es posible que se esté aplicando una cantidad de agua superior o inferior a la que en realidad se requiere (Gomes, 2013). El suministro de agua es uno de los factores determinantes para el desarrollo y producción de las plantas, y su requerimiento hídrico depende de las condiciones climáticas de la zona, suelo, tipo de cultivo, manejo y etapa en la que se encuentre (Gómez et al., 2010). La aplicación del riego de manera oportuna y suficiente es algo fundamental en la producción de plántulas de cualquier especie comercial, puesto que la falta o exceso de agua puede comprometer tanto el crecimiento y desarrollo de las mismas como su productividad en campo (Oliveira, 2012).

Para producir plántulas forestales de calidad se deben buscar manejos hídricos que minimicen los negativos impactos ambientales, económicos y sociales causados por el mal manejo del agua (Gomes, 2013). En este contexto, se planteó evaluar el efecto de cuatro láminas de riego sobre las variables de crecimiento de Gmelina arborea, con el objetivo de conocer su requerimiento hídrico en la etapa de vivero.

\section{MATERIALES Y MÉTODOS}

El trabajo experimental se llevó a cabo bajo condiciones controladas en la Universidad de Córdoba (Montería, Córdoba - Colombia), ubicada a $8^{\circ} 48^{\prime}$ Latitud Norte y $75^{\circ} 52^{\prime}$ Longitud Oeste, con una elevación de 15 m.s.n.m. El área de entorno presenta una precipitación media anual de $1200 \mathrm{~mm}$, temperatura promedio de $28^{\circ} \mathrm{C}$, brillo solar de 1800 horas luz/año y una humedad relativa del $84 \%$. La temperatura promedio en el sitio de investigación es de $34{ }^{\circ} \mathrm{C}$ y humedad relativa de $65 \%$, la evapotranspiración de referencia (ETo) máxima en el periodo del estudio fue $4 \mathrm{~mm} \cdot \mathrm{dí}^{-1}$.

Se realizó un diseño completamente aleatorizado con 4 tratamientos y 4 repeticiones, correspondientes a cuatro coeficientes de consumo (Kc: 0,6, 0,8, 1,2, 1,4). Estos tratamientos fueron transformados en láminas de riego de 2,4 mm.día ${ }^{-1}$, 3,2 mm.día ${ }^{-1}$ 4,8 mm.día ${ }^{-1}$ y 5,6 mm.día ${ }^{-1}$, respectivamente al utilizar la Ecuación $1, \mathrm{y}$ fueron aplicados mediante un sistema de riego por goteo durante un periodo de 45 días.

$U C=K c \times$ ETomax

UC: Uso consuntivo o evapotranspiración del cultivo (mm.día $\left.{ }^{-1}\right)$

Kc: Coeficiente del cultivo

ETomax: evapotranspiración de referencia máxima (mm.día $\left.{ }^{-1}\right)$

Cada tratamiento contó con 40 unidades experimentales conformado por cuatro hileras (repeticiones) de 10 plantas distanciadas $20 \mathrm{~cm}$ y $30 \mathrm{~cm}$ entre hileras. El área total de experimento fue de $15 \mathrm{~m}^{2} \mathrm{y}$ en total se utilizaron 160 plantas.

Para la investigación se utilizó un material multiclonal proveniente de la empresa 3F Kanguroid, las plántulas vinieron enraizadas con 2 pares de hojas y longitud promedio de $8 \mathrm{~cm}$. Estas fueron establecidas en bolsas de polietileno de diámetro de $22 \mathrm{~cm}$ de ancho por $35 \mathrm{~cm}$ longitud, calibre 3 , en un sustrato 1,5:1:0,5 (arena, suelo, materia orgánica) que fue previamente desinfectado. El riego en la primera semana se hizo uniforme, con el fin de aclimatar las plantas y dejarlas en condiciones iguales. La aplicación de los tratamientos se inició una 
semana después de que fueran trasplantadas en las bolsas, momento en que las plantas presentaban 2 pares de hojas y una longitud promedio de $10 \mathrm{~cm}$.

La medición de las variables se realizó 35 días después del inicio de los tratamientos (DDIT), y en total se hicieron 6 muestreos destructivos cada 2 días, utilizando 4 plantas por tratamiento. Las variables medidas fueron: altura de la planta (AP) en cm, área foliar (AF) en $\mathrm{cm}^{2}$, masa seca de la hoja (MSH) en gramos, masa seca del tallo (MST) en gramos, masa seca de la raíz (MSR) en gramos, masa seca total o biomasa total (BMT) en gramos.

La altura de la planta se tomó con la ayuda de un flexómetro, midiéndose la AP desde la base del tallo hasta el ápice de la planta. El AF se calculó con la ayuda del software Determinador Digital de Áreas (DDA). Las variables de masa seca de hoja, tallo y raíz (MSH, MST, MSR) se obtuvieron después de haber sometido cada parte a un proceso de secamiento en una estufa a razón de $80^{\circ} \mathrm{C}$ durante 48 horas, y la biomasa total (BMT) es la suma de las masas secas de las partes de la planta. Las medidas indirectas de crecimiento (TAC, TRC, RAF, AFE,) se calcularon mediante las Ecuaciones $2-5$, respectivamente.

$$
T A C=\frac{M S_{2}-M S_{1}}{T_{2}-T_{1}}
$$

TAC: Tasa absoluta de crecimiento (g.día ${ }^{-1}$ )

$\mathrm{MS}_{1}$ : Masa seca del muestreo anterior (g)

$\mathrm{MS}_{2}$ : Masa seca del siguiente muestreo (g)

$\mathrm{T}_{1}$ : Día del muestreo anterior

$\mathrm{T}_{2}$ : Día del muestreo siguiente

$T R C=(\ln M S f-\ln M S i) /(T f-T i)$

TRC: Tasa relativa de crecimiento $\left(\mathrm{g} \cdot \mathrm{g}^{-1} \cdot \mathrm{dí}^{-1}\right)$

lnMSi: Logaritmo natural de la masa seca muestreo anterior $(\mathrm{g})$

lnMSf: Logaritmo natural de la masa seca muestreo siguiente $(\mathrm{g})$

Ti: Día del muestreo anterior

Tf: Día del muestreo siguiente

$A F E=\frac{A F}{M S H}$

AFE: Área foliar efectiva $\left(\mathrm{m}^{2} \cdot \mathrm{g}^{-1}\right)$

AF: Área foliar $\left(\mathrm{m}^{2}\right)$

MSH: Masa seca de la hoja (g)
$R A F=\frac{A F t}{B M T}$

RAF: Relación de área foliar $\left(\mathrm{cm}^{2} \cdot \mathrm{g}^{-1}\right)$

AFt: Área foliar total $\left(\mathrm{cm}^{2}\right)$

BMT: Biomasa seca total $(\mathrm{g})$

Los datos obtenidos fueron tabulados en el programa Excel, versión 2010, y procesados mediante el programa SAS, versión 9.2; las gráficas se realizaron con el programa SigmaPlot versión 10.0.

\section{RESULTADOS Y DISCUSIONES}

\subsection{Altura de planta}

Según el análisis estadístico, se encontraron diferencias significativas $(\mathrm{P}<0,05)$ entre los tratamientos de láminas de riego, con un coeficiente de variación de 10,62. La mayor altura fue de $39,825 \mathrm{~cm}$ y se presentó con la menor lámina de riego, mientras que en la lámina de 5,6 mm.día ${ }^{-1}$, la mayor de todas, se presentaron los menores valores promedios de altura de planta. La Figura 1 muestra la tendencia de la altura de las plantas con relación a la lámina de riego, exponiendo una función cuadrática negativa, la cual indica que, a medida que se incrementa la lámina de riego, la altura de la planta tiende a disminuir linealmente $5,26 \mathrm{~cm}$ por cada vez que se aumenta 1 (un) mm de riego al día.

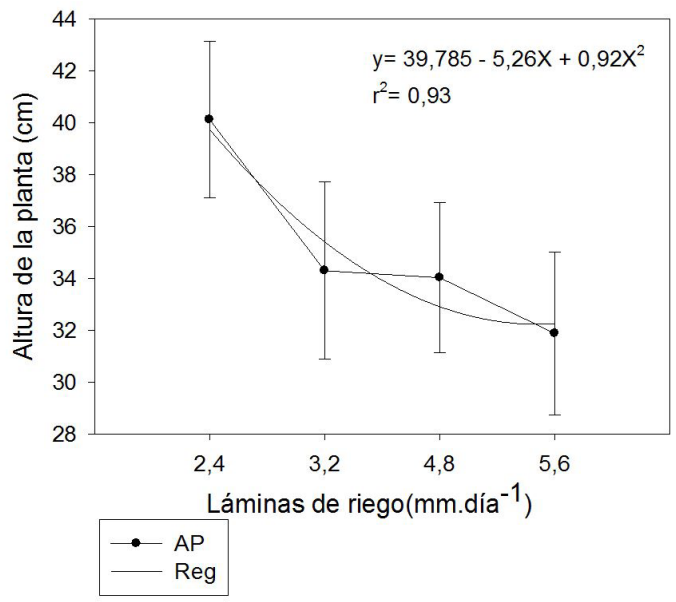

Figura 1. Efecto de las láminas de riego aplicadas sobre la altura de planta (AP) en Gmelina arborea.

Figure 1. Effect of irrigation levels applied on plant height $(\mathrm{PH})$ in Gmelina arborea. 
Los resultados descritos coinciden con los reportados por Martinelli (2012), quien encontró mayores alturas en plantas de Hymenaea courbaril, regadas con las menores láminas y frecuencia de riego de dos veces al día. Por su parte Rawat et al. (1985), trabajando con Eucaliptus terraticornis bajo diferentes niveles de humedad, concluye que el crecimiento en altura no fue proporcional al aumento de la humedad. Fox \& Montague (2009) afirman que el mayor crecimiento de las plantas no siempre se asocia con láminas altas de riego, por lo cual se podrían economizar grandes cantidades de agua durante el crecimiento de las plántulas en vivero. De esta manera se puede inferir que, para aumentar la altura de la planta de Gmelina arborea en la etapa de vivero, no son necesarias láminas superiores de 2,4 mm.día ${ }^{-1}$, ya que la altura de la planta depende de la disponibilidad de agua en un cierto punto, que es en el que las raíces no pueden captar suficiente oxígeno por el exceso hídrico y disminuyen su velocidad de crecimiento (Salisbury \& Ross, 1994).

\section{2. Área Foliar (AF)}

A los 45 días después del inicio de los tratamientos (DDIT) se encontraron diferencias altamente significativas $(\mathrm{P}<0,01)$ entre los tratamientos para la variable área foliar (AF). La prueba del rango estudentizado de Tukey mostró que la lámina de 2,4 mm.día ${ }^{-1}$ fue estadísticamente diferente al resto de los tratamientos y presentó los valores promedios más altos de AF $\left(1115,38 \mathrm{~cm}^{2}\right)$. En la Figura 2 se observa que existe una

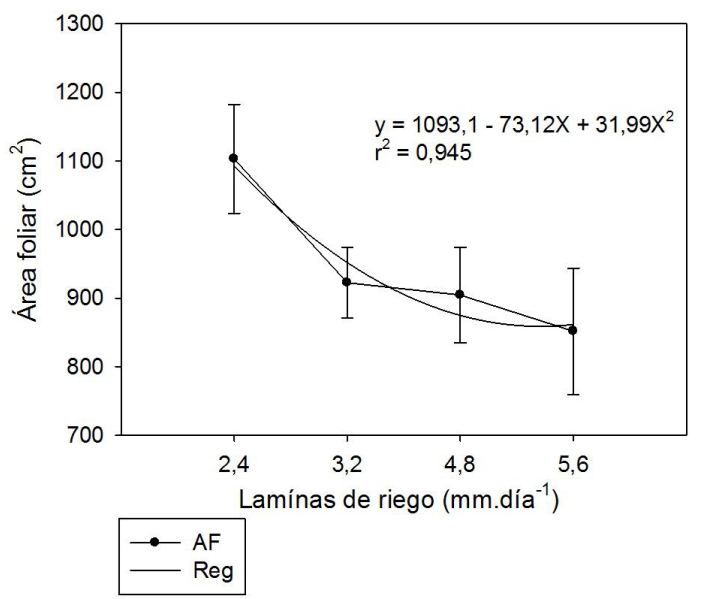

Figura 2. Efecto de las láminas de riego aplicadas sobre el área foliar (AF) en Gmelina arborea.

Figure 2. Effect of irrigation levels applied to the leaf area (LA) in Gmelina arborea. función cuadrática negativa entre la lámina de riego aplicada y la variable AF con un $r^{2}=0,94$, indicando que por cada milímetro de riego a partir de $2,4 \mathrm{~mm}$, el área foliar decrece $173,12 \mathrm{~cm}^{2} \cdot \mathrm{dia}^{-1}$.

En este estudio se observó que el aumento de cantidad de agua aplicada va unido al decrecimiento del área foliar. Dichos resultados se asemejan a los de Falcão (2012), quien estudiando la producción de plántulas de Parapiptadenia rigida en diferentes sustratos y láminas de riego, encontró mayor área foliar cuando aplicó la menor lámina (4 mm.día $\left.{ }^{-1}\right)$ independientemente del sustrato. Según Larcher (2006), la disminución en el área foliar puede estar asociada con la reducción en el tamaño de las hojas o en su producción, y, por lo tanto, la zona de captación de la luz, las reacciones fotosintéticas y el intercambio gaseoso se ven severamente influenciados. Cairo (1995) y Lovelock et al. (1998), estudiando especies forestales, determinaron que el área foliar es extremadamente afectada por la cantidad de agua disponible en el suelo. En la presente investigación los valores más altos de área foliar se registraron con la menor lámina aplicada $\left(2,4 \mathrm{~mm} \cdot \mathrm{día}^{-1}\right)$, lo cual indica que la especie Gmelina arborea demanda una baja cantidad de agua en la etapa de vivero.

\subsection{Masa Seca de Hojas (MSH).}

Los valores promedios más altos para esta variable se obtuvieron con 2,4 mm.día ${ }^{-1}$ (3,9 g), seguido por 3,2 mm.día ${ }^{-1}$ (3,65 g), 4,8 mm.día ${ }^{-1}$ (3,53 g) y 5,6 mm.día ${ }^{-1}$ $(3,3 \mathrm{~g})$, respectivamente. La Figura 3 muestra que a medida que se incrementa la lámina de riego tiende a disminuir el valor de la biomasa en las hojas.

Falcão (2012), estudiando la producción de plántulas Parapiptadenia rigida en diferentes sustratos y láminas de riego, encontró el mayor porcentaje de masa seca de la hoja cuando aplicó la menor cantidad de agua. Dichos resultados se asemejan a los de la presente investigación. Sin embargo, la misma autora, estudiando la especie Lueheae divaricata, observó un aumento en la materia seca de la hoja conforme aumentaba la lámina de riego. Por otro lado, Santiago et al. (2002) y Cabral et al. (2004) encontraron en plántulas de ipê amarelo (Tabebuia chrysotricha) que se redujo notablemente el peso seco de las hojas cuando se aplicó una menor cantidad de agua. 


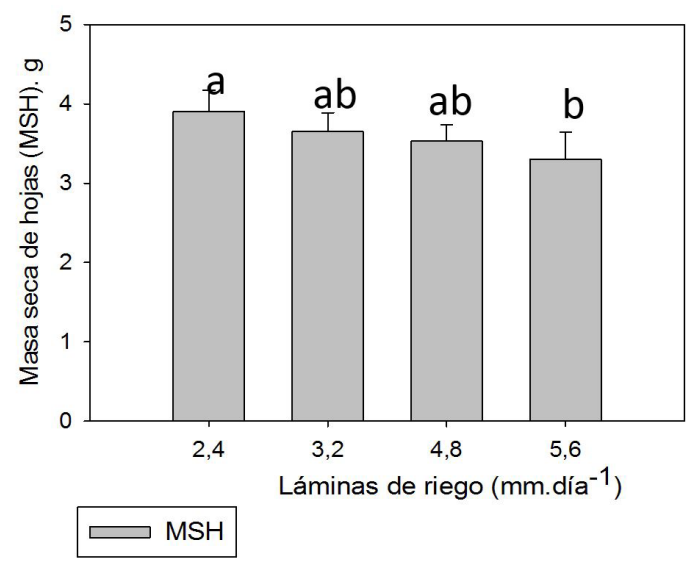

Figura 3. Efecto de las láminas de riego aplicadas sobre la masa seca de las hojas (MSH) en Gmelina arborea. Letras iguales no representan diferencias estadísticas.

Figure 3. Effect of irrigation levels applied to the dry leaf mass (LM) in Gmelina arborea. Equal letters do not represent statistical differences.

\subsection{Masa Seca del Tallo (MST)}

El análisis de varianza mostró diferencias altamente significativas $(\mathrm{P}<0,01)$ entre los tratamientos para la variable MST. La Figura 4 muestra que T1 (2,4 mm.día $\left.{ }^{-1}\right)$ presentó el mejor valor de MST, mientras que T4 (5,6 mm.día $\left.{ }^{-1}\right)$ mostró el menor valor, de lo cual se puede deducir que la masa seca del tallo tiende a disminuir a medida que se incrementa la lámina de riego.

Silva et al. (2002) observó que no hubo un efecto significativo en MST para los tratamientos de niveles de riego y aplicaciones de potasio en plantas de E. grandis. Sin embargo se puede afirmar que el peso seco de las plantas se reduce cuando hay exceso o déficit de humedad en el suelo, lo que en parte se debe a la reducción de la materia seca del tallo; esto puede ser porque la capacidad fotosintética se inhibe bajo condiciones de exceso o déficit hídrico (Casierra \& Gómez, 2008). Además, cuando el volumen de agua excede la capacidad de la retención del sustrato se produce una lixiviación de los nutrientes (Thomas \& Perry, 1980). En esta investigación las láminas superiores a 2,4mm.día ${ }^{-1}$ pudieron generar esta condición.

\subsection{Masa Seca De La Raíz (MSR)}

Los valores promedios más altos para esta variable se obtuvieron de 2,4 mm.día ${ }^{-1}$ (3,23 g) seguido de 3,2 mm.día ${ }^{-1}(2,83 g), 4,8$ mm.día ${ }^{-1}(2,75 g)$ y 5,6 mm.día ${ }^{-1}$

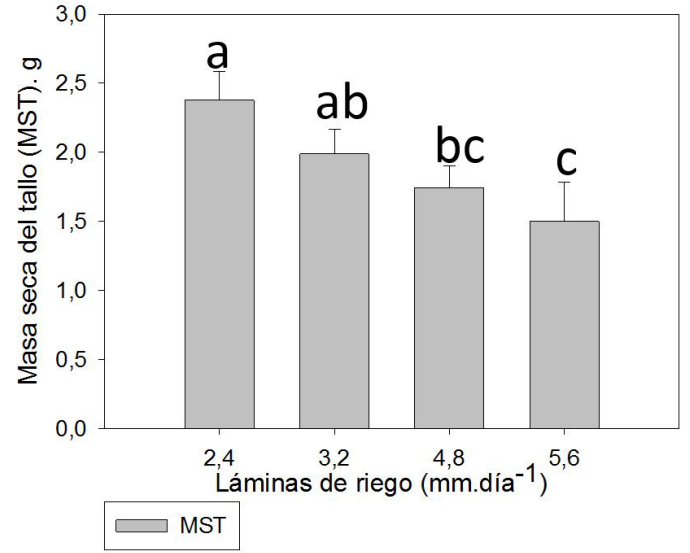

Figura 4. Efecto de las láminas de riego aplicadas sobre la masa seca del tallo (MST) en Gmelina arborea. Letras iguales no representan diferencias estadísticas.

Figure 4. Effect of irrigation levels applied to the stem dry weight (SDW) in Gmelina arborea. Equal letters do not represent statistical differences.

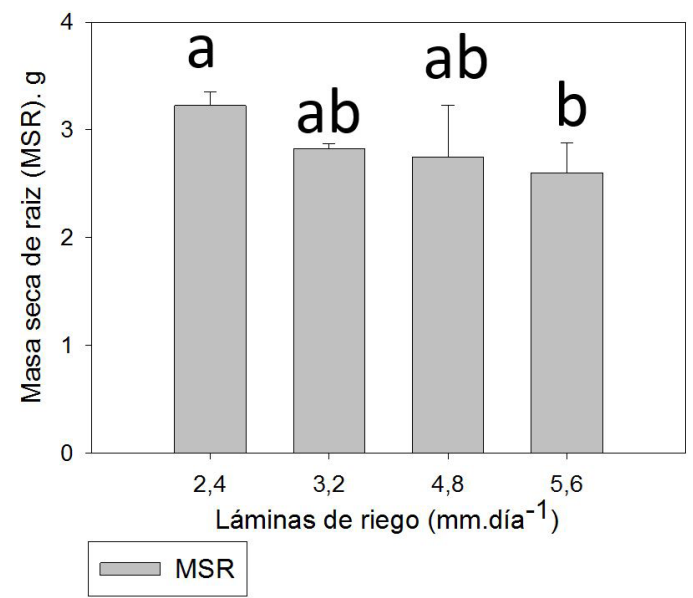

Figura 5. Efecto de las láminas de riego aplicadas sobre la masa seca de la raíz (MSR) en Gmelina arborea. Letras iguales no representan diferencias estadísticas.

Figure 5. Effect of water depths applied on the root dry weight (RDW) in Gmelina arborea. Equal letters do not represent statistical differences.

$(2,60 \mathrm{~g})$, respectivamente. En la Figura 5, se observa que el mayor valor promedio de MSR se encontró con la lámina de $2,4 \mathrm{~mm}$.día ${ }^{-1}$ y el menor valor con el T4 (5,6 mm.día $\left.{ }^{-1}\right)$, con una tendencia de disminución de la biomasa de raíces a medida que se incrementa la lámina de riego.

Martinelli (2012), evaluando la calidad morfológica en plántulas de Peltophorum dubium bajo diferentes 
manejos de agua, encontró el mayor valor de masa seca de raíz en la menor lámina de riego aplicada. También reportó que la menor lámina de riego presentó el mayor porcentaje de plantas con un sistema radicular de mejor calidad para ir a campo, unos resultados que se asemejan a los de la presente investigación. Por su parte Lopes et al. (2005), trabajando con Eucalyptus grandis en diferentes láminas de riego, concluye que hay un aumento gravimétrico de materia seca radicular conforme se aumenta la lámina de riego. Asimismo, Santiago et al. (2002) y Cabral et al. (2004), reportaron de sus investigaciones que los valores más altos de masa seca radicular se encuentran cuando se aplica una mayor cantidad de agua, lo cual no concuerda con el presente estudio.

\subsection{Biomasa Seca Total (BMT)}

El análisis de varianza mostró diferencias altamente significativas $(\mathrm{P}<0,01)$ entre los tratamientos para la variable BMT, con un coeficiente de variación de 6,25 . La Figura 6 muestra la biomasa seca total de las plantas Gmelina arborea con relación a la lámina de riego aplicada, y se observa que la biomasa seca total tiende a disminuir a medida que se incrementa la lámina de riego.

Myers \& Landsberg (1989), estudiando las plántulas de E. maculata y E. brockwayi bajo diferentes niveles

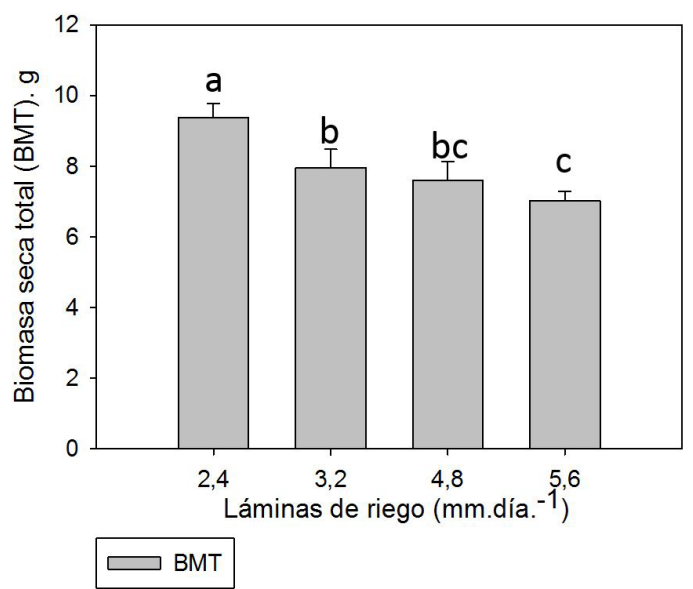

Figura 6. Efecto de las láminas de riego aplicadas sobre la biomasa seca total (BMT) en plantas de Gmelina arborea. Letras iguales no representan diferencias estadísticas.

Figure 6. Effect of applied water depths on the total dry biomass (TDB) in Gmelina arborea. Equal letters do not represent statistical differences. de riego, observaron el aumento de materia seca en proporción con el menor régimen hídrico, con unos resultados semejantes a los del presente estudio. Silva (1998), en su trabajo con plántulas de E. grandis, observó que un régimen hídrico severo genera un menor contenido de biomasa seca total. En esta investigación se encontró que al aumentar la lámina de riego disminuye la $\mathrm{BMT}$; esta disminución puede estar relacionada con una reducción del oxígeno en la zona radicular de las plantas. Diversos autores han documentado las consecuencias de las deficiencias de oxígeno en el suelo para plantas no adaptadas, debido a la limitada difusión de este gas bajo el agua, lo que dificulta el intercambio gaseoso necesario para los procesos críticos de fotosíntesis y respiración aeróbica (Sasidharan \& Voesenek, 2015). La baja difusión de gases, asociada a condiciones de saturación de agua en suelo, impone un cambio drástico en la composición interna de los órganos de las plantas y de sus respuestas bioquímicas, como lo indica Herrera (2013) en su trabajo con árboles tropicales de América del sur; uno de los primeros cambios que presentan todas las células inundadas es una rápida acumulación del etileno volátil, una hormona generalmente asociada con la senescencia de tejidos vegetales, entre otros efectos. Aunque no fueron medidas en este experimento, existe otro factor determinante en las respuestas del decrecimiento de la biomasa vegetal cuando se aplican láminas altas de agua, hasta alcanzar niveles de saturación de humedad en el suelo: es la producción de especies reactivas de oxígeno (Steffens et al., 2013; Pucciariello et al., 2014), que se presentan en condiciones de estrés y que, si no son removidas rápidamente, tienen efectos negativos sobre eficiencia metabólica de la membrana celular; probablemente en este caso, para establecer sus mecanismos de defensa antioxidante, las plántulas invierten una gran cantidad de energía, en detrimento de la construcción de la biomasa para el crecimiento.

\subsection{Indices de crecimiento}

El comportamiento de los índices de crecimiento (TRC, TAC, RAF y AFE) para la especie Gmelina arborea en etapa de vivero se muestra en la Figura 7, donde se observa un aumento en biomasa a lo largo de los días del experimento, hasta que finalmente la ganancia en masa tiende a disminuir. 

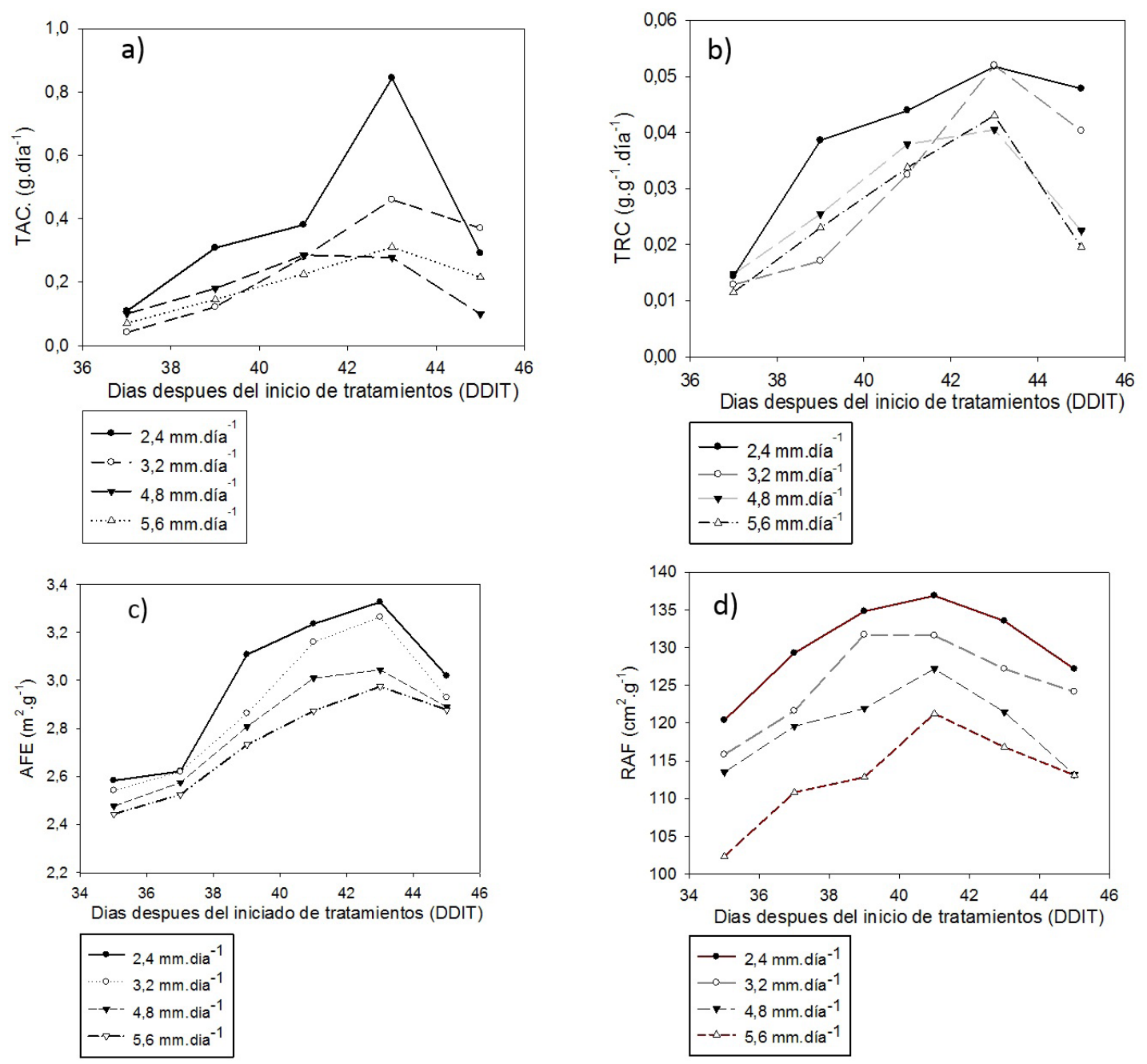

Figura 7. Comportamiento de los índices de crecimiento (a) TAC, (b) TRC, (c) AFE, (d) RAF en Gmelina arborea sometida a diferentes láminas de riego.

Figure 7. Behavior growth rates (a) AGR, (b) TRC, (c) AFE, (d) RLA in Gmelina arborea exposed to various irrigation levels.

Se observó que la tasa absoluta de crecimiento (TAC) aumentó en función del tiempo, pero a partir del día 43 decreció. Mithorphe \& Moorby (1982) afirman que la TAC está en función de la cantidad de material de crecimiento presente y de la tasa de crecimiento per se, motivo por el cual, al inicio del desarrollo existe un periodo en que esta función del crecimiento es cada vez mayor, posteriormente se mantiene casi constante y luego comienza a disminuir. La tasa relativa de crecimiento (TRC), según Hunt (1982) y Gardner et al. (1990), representa la eficiencia de la planta para producir nuevo material en un tiempo determinado expresado en grados de materia seca ganados por gramo de materia seca existente por unidad de tiempo $\left(\mathrm{g} \cdot \mathrm{g}^{-1} \cdot \mathrm{dia}^{-1}\right)$.

Por otro lado, se considera la TRC como un índice de eficiencia en la producción de peso seco de las plantas; es constante durante su fase inicial y a medida que aumenta la edad de la planta esta va disminuyendo (Silvori \& Montaldi, 1980). Se observó inicialmente un incremento progresivo de la TRC, pero se presentó una disminución de este índice a los 43 DDIT, lo que indica que a partir de este día el tejido es menos eficiente para producir biomasa.

Hunt (1982) afirma que la relación de área foliar (RAF) indica la cantidad de área foliar producida 
con relación al peso seco total de la planta, mientras que el área foliar efectiva (AFE) es el componente morfológico y anatómico de la relación de área foliar, debido a que relaciona la superficie de la hoja con su propia materia seca. De acuerdo a los resultados presentados se encontró que las plantas de melina con una baja lámina de agua fueron más eficientes desde el punto de vista de fotosíntesis.

Por todo lo anterior, se determinó que las plantas con mayor crecimiento y desarrollo fueron las que recibieron una lámina de 2,4 mm.día ${ }^{-1}$. Wild (1992) afirma que el crecimiento es el aumento del tamaño de la planta, el cual puede ser en materia seca o en dimensiones como consecuencia de la formación de nuevas células, la expansión de las células constituyentes y el almacenamiento de asimilados. Pero, independientemente de la lámina aplicada, se observó que la TAC, la TRC y la AFE disminuyen a partir del día 43 después del inicio de tratamientos (DDIT); asimismo la RAF se redujo a los 41 dias, lo que indica que las plantas en este punto ya no están creciendo significativamente y que ese es el tiempo adecuado para el trasplante.

\subsection{Determinación del coeficiente de cultivo (Kc) para Melina (Gmelina arborea) en etapa de vivero}

De acuerdo con los resultados obtenidos en las variables e índices de crecimiento y desarrollo, se determinó que el coeficiente de cultivo $(\mathrm{Kc})$ es 0,6 para Gmelina arborea en la etapa de vivero. Este Kc se considera un valor medio, lo cual indica que los requerimientos hídricos para la especie forestal en estudio en esta fase no son altos y puede haber un gran ahorro de agua si dicho Kc se tiene en cuenta. Santos (2007), estudiando las necesidades de agua en plantas de Eucaliptus, recomienda la aplicación de una lámina de riego aproximada al 257\% de la evapotranspiración de referencia. Gómez et al. (2010) señala que el suministro de agua es uno de los factores determinantes para el desarrollo y producción de las plantas, y su requerimiento hídrico depende de las condiciones climáticas de la zona, suelo, tipo de cultivo, manejo y etapa en la que se encuentre. En general, existen pocos trabajos sobre requerimientos hídricos de Gmelina arborea en esta fase de crecimiento.

\section{CONCLUSIONES}

De las dosis de agua evaluadas en plantas de melina en la etapa de vivero, la lámina de riego de 2,4 mm.día ${ }^{-1}$ presentó la mayor acumulación de biomasa seca total, mayor altura y mayor área foliar.

Las diferencias encontradas para las variables estudiadas, con respecto a los tratamientos aplicados, fueron determinantes para la elección del coeficiente de cultivo Kc de la especie. Este coeficiente tiene un valor de 0,6 , considerándoselo como valor medio, lo que indica que el consumo de agua de esta planta en vivero es bajo, y para las condiciones la zona de estudio es de 2,4 mm.día ${ }^{-1}$.

Cuando se aplican láminas de riego superiores a $2.4 \mathrm{~mm} \cdot$ día $^{-1}$ el crecimiento en Gmelina arborea en esta fase de vivero tiende a disminuir. Esto puede indicar que láminas por encima de este valor ocasionan exceso de humedad en el sustrato y la disminución de la cantidad de oxigeno, lo cual genera alteraciones en la fisiología de la planta.

Independientemente de la lámina aplicada, entre los 41 y 43 DDIT se observó la disminución en los índices de crecimiento. Por tanto, se considera que este es el punto máximo en el cual las plantas de melina deben ser trasplantadas a campo y, así, brindarles otras condiciones.

\section{ESTADO DE LA SUMISIÓN}

Recibido: 6 sep., 2016

Aceptado: 16 sep., 2016

AUTOR(ES) PARA LA CORRESPONDENCIA

\section{Teobaldis Mercado}

Facultad de Ciencias Agricols, Universidad de Córdoba, Carrera 6, no. 76, 103, Montería, Córdoba, Colombia e-mail: teobaldis@gmail.com

\section{REFERENCIAS}

Cabral EL, Barbosa DC, Simabukuro EA. Crescimento de plantas jovens de Tabebuia áurea (Marsh) Benth. \& Hook. F. ex s. moore submetida a estresse hídrico. Acta Botanica Brasílica 2004; 18(2): 241-251. http://dx.doi. org/10.1590/S0102-33062004000200004. 
Cairo PAR. Curso básico de relações hídricas de plantas. Vitória da Conquista: Universidade Estadual do Sudoeste da Bahia; 1995. 32 p.

Casierra F, Gómez N. Crecimiento foliar y radical en plantas de fique (Furcraea castilla y F. macrophylla) bajo estrés por encharcamiento. Agronomía Colombiana 2008; 26(3): 381-388.

Falcão, DA. Produção de mudas de Parapiptadenia rigida (Benth.) Brenan E Luehea divaricata Mart. Et Zucc. em diferentes substratos e lâminas de irrigação [disertación]. Santa Maria: Universidade Federal de Santa Maria; 2012.

Fox L, Montague T. Influence of irrigation regime on growth of select field-grown tree species in a semi-arid climate. Journal of Environmental Horticulture 2009; 27(3): 134-138.

Gardner FP, Brent PR, Mitchell RL. Physiology of crop plants. 2nd ed. Ames: Iowa State University; 1990.

Gatti K, Suarez I, Espitia M, Tobar D. Producción de plántulas forestales de Tectona grandis Linn F, Acacia mangium Willdy Gmelina arborea Roxb por miniestacas. Córdoba: Universidad de Córdoba; 2012.

Gomes RB. Manejo hídrico sobre o desenvolvimento e a qualidade de mudas florestais nativas em ambientes protegidos [disertación]. Botucatu: Faculdade de Ciências Agronômicas, Universidade Estadual Paulista; 2013.

Gómez A, Rojas H, Vallejo A, Estrada E. Determinación del requerimiento hídrico del pimentón en el municipio de candelaria, departamento del valle del cauca. Acta Agronomica 2010; 59(4): 442-448.

Herrera A. Responses to flooding of plant water relations and leaf gas exchange in tropical tolerant trees of a black-water wetland. Frontiers in Plant Science 2013; 4(150): 1-12. PMid:23641246. http://dx.doi.org/10.3389/ fpls.2013.00106.

Hunt R. Plant growth curves: the functional approach to plant grow analysis. London: Edwar Arnold; 1982. 248 p.

Larcher W. Ecofisiologia vegetal. São Carlos: Rima; 2006. $531 \mathrm{p}$.

Lopes JL, Guerrini IA, Saad JC. Efeitos de lâminas de irrigação na produção de mudas de Eucalyptus grandis W. (Hill Ex. Maiden) em substrato de fibra de coco. Revista Irriga 2005; 10(2): 123-13.

Lovelock CE, Winter K, Mersits R, Popp, M. Responses of communities of tropical tree species to elevated CO 2 in a forest clearing. Oecologia 1998; 116(1-2): 207-218. http://dx.doi.org/10.1007/s004420050581.

Mariño Y, Rodríguez M. El punto de marchitez permanente (pmp) en melina (Gmelina arborea. Roxb) para la costa caribe colombiana ¿una característica para la selección de clones? Revista Corpoica: Ciencia y Tecnología Agropecuaria 2010; 11(2): 116-121. http:// dx.doi.org/10.21930/rcta.vol11_num2_art:201.
Martinelli. L. Produção de mudas nativas sob diferentes manejos hídricos [disertación]. Botucatu: Faculdade de Ciências Agronómicas, Universidade Estadual Paulista; 2012.

Mithorphe FL, Moorby J. An introduction to crop physiology. Cambridge: Cambridge University Press; 1982. 202 p.

Murillo O, Espitia M, Castillo C. Fuentes semilleras para la producción forestal. Córdoba: Universidad de Córdoba; 2012.

Myers BJ, Landsberg JJ. Drought and seedling growth of two eucalypt species from contrasting habitats. Tree Physiology 1989; 5(1): 207-218. PMid:14972988. http:// dx.doi.org/10.1093/treephys/5.2.207.

Oliveira A. Otimazação do uso da água na produção de mudas clonais de eucaliptus [disertación]. Viçosa: Programa de Pós-graduação em Meteorología Agrícola, Universidade Federal de Viçosa; 2012.

Prieto J, Domínguez P, Cornejo E, Návar J. Efecto del envase y del riego en vivero en el establecimiento de pinus cooperi blanco en dos condiciones de sitio. Madera y Bosques 2007; 13(1): 79-97.

Pucciariello C, Voesenek L, Perata P, Sasidharan R. Plant responses to flooding. Frontiers in Plant Science 2014; 5: 226. PMid:24904621. http://dx.doi.org/10.3389/ fpls.2014.00226.

Rawat PS, Gupta BB, Rawat JS. Tranpiration as affected by soil moisture in Eucalytus tereticornis seedlings. Indian Forester 1985; 11(1): 35-39.

Salisbury F, Ross C. Fisiología vegetal. 4. ed. México, D.F.: Grupo Editorial Iberoamérica S.A.; 1994. 759 p.

Santiago MP, Nogueira MC, López EC. Crescimento de plantas jovens de Mimosa caesalpiniifolia Benth cultivada sob estresse hídrico. Revista Ecossistema 2002; 26(1): 23-30.

Santos RS. Analise do uso de agua en unidades de produçao de mudas de eucalipto [disertación].Viçosa: Universidade Federal de Viçosa; 2007.

Sasidharan R, Voesenek L. Ethylene-mediated acclimations to flooding stress. Plant Physiology 2015; 169(1): 3-12. PMid:25897003. http://dx.doi.org/10.1104/pp.15.00387.

Silva MR. Caracterização morfológica, fisiológica e nutricional de mudas de Eucaliptus grandis Hill ex. Maiden submetidas a diferentes níveis de estresse hídrico durante la fase de rustificação [disertación]. Curitiba: Universidade Federal do Paraná; 1998.

Silva RBG, Simões D, Silva MR. Qualidade de mudas clonais de Eucalyptus urophylla x E. grandis em função do substrato. Revista Brasileira de Engenharia Agrícola e Ambiental 2012; 16(3): 297-302. http://dx.doi.org/10.1590/ S1415-43662012000300010.

Silva SRS, Demuner AJ, Barbosa LCA, Casali VWD, Nascimento EA, Pinheiro AL. Efeito do estresse hídrico sobre características de crescimento e produção de óleo 
essencial de Melaleuca alternifólia Cheel. Acta Scientiarum 2002; 24(5): 1363-1368.

Silvori ME, Montaldi RE. Fisiología vegetal. Buenos Aires: Ed. Hemisferio Sur S.A.; 1980. 106 p.

Steffens B, Steffen-Heins A, Sauter M. Reactive oxygen species mediate growth and death in submerged plants. Frontiers in Plant Science 2013; 4(179): 1-7. PMid:23761805. http://dx.doi.org/10.3389/fpls.2013.00179.
Taiz L, Zeiger E. Fisiologia vegetal. 3. ed. Porto Alegre: Artmed; 2004.719 p.

Thomas S, Perry FB. Ammonium nitrogen accumulation and leaching from an all pine bark medium. HortScience 1980; 15(6): 824-825.

Wild A. Condiciones del suelo y desarrollo de las plantas. Madrid: Ed. Mundiprensa; 1992. 1045 p. 